\title{
No simpler than mammals: axon and dendrite regeneration in Drosophila
}

\author{
Homaira Nawabi, Katherine Zukor, and Zhigang $\mathrm{He}^{1}$ \\ F.M. Kirby Neurobiology Center, Children's Hospital Boston, Boston, Massachusetts 02115, USA
}

Despite important progress made in understanding the mechanisms of axon regeneration, how a neuron responds to an injury and makes a regenerative decision remains unclear. In this issue of Genes \& Development, Song and colleagues (pp. 1612-1625) investigate axonal and dendritic regeneration in the Drosophila peripheral nervous system (PNS). With some mechanisms shared with mammals, this study reveals surprisingly complicated regenerative responses in terms of cell type, developmental stage, and mechanism specificity. With forward genetic potential, such invertebrates should be powerful in dissecting the cellular and molecular control of neuronal repair.

In textbooks, regeneration failure has been generally described as an issue of the mammalian CNS. As a result, the majority of studies have been performed in mammals or mammalian neuronal cultures. Through these, it has been established that both neuron-intrinsic growth ability and the extracellular environment control the process of nerve regeneration (Yiu and He 2006; Fitch and Silver 2008; Schwab 2010; Liu et al. 2011; Bradke et al. 2012). Importantly, the molecular identities of such extrinsic and intrinsic contributors are starting to be revealed. However, because of technical limitations, mammalian models are not easily amenable to some of the powerful manipulations, such as forward genetic studies and in vivo imaging, that are feasible in lower organisms. It has been assumed that, like the mammalian peripheral nervous system (PNS), lower organisms can always regenerate their axons spontaneously. These assumptions, however, have not been formally tested in many cases, and a new example to the contrary is presented in the study by Song et al. (2012) in this issue of Genes \& Development. In addition to revealing important cellular and molecular insights into the complexity of axon and even dendrite regeneration, this study highlights the potential

[Keywords: CNS; Pten; bantam; dendritic arborization neuron; microRNA; regeneration]

${ }^{1}$ Corresponding author

E-mail zhigang.he@childrens.harvard.edu

Article is online at http://www.genesdev.org/cgi/doi/10.1101/gad. 198150.112 . for injury models in invertebrates and lower vertebrates to complement those in mammals.

\section{Model systems for regeneration studies}

Albeit infrequently, lower vertebrates and invertebrates have been used for some time in neuronal injury studies, and these studies demonstrate the potential lower organisms have for revealing insights into nerve regeneration (Spira et al. 2003; Martin et al. 2010; Chen et al. 2011). Because of their large size and reliable growth ability, neurons from Aplysia californica and squid have been a powerful model in studying how neurites respond to injury in vivo (Krause et al. 1994; Spira et al. 2003; Erez et al. 2007). With the development of precise femtosecond laser-assisted lesion methods (Yanik et al. 2004), it is also now possible to monitor the live injury responses of single axons in lower organisms that have much smaller neurons. Using this method in transgenically labeled fluorescent axons in Caenorhabditis elegans, it has been shown that axons from a variety of neurons, such as the GABAergic motor neurons and mechanosensory neurons, are able to regenerate after injury. Combining this with powerful genetic screens to find mutations that disrupt this regeneration, several important signaling pathways and molecules controlling the process of axon regeneration have been identified (Hammarlund et al. 2009; Yan et al. 2009; Bejiani and Hammarlund 2012). Furthermore, now that laser-assisted axotomy can be facilitated with customized microfluidic devices, C. elegans is poised for high-throughput genetic and pharmacological studies (Guo et al. 2008; Ghannad-Rezaie et al. 2012). Such axotomy procedures have also been used in other model organisms, such as zebrafish and Drosophila (Sugimura et al. 2003; O'Brien et al. 2009; Martin et al. 2010). In general, these studies are consistent with the dogma that axons in these lower organisms are all capable of regeneration. Recent results, however, demonstrate that this is not always the case.

\section{Cell type-specific axon regeneration in Drosophila}

Using such laser-assisted lesioning, Song et al. (2012) examined the regenerative responses to axotomy as well as dendriotomy in Drosophila's sensory dendritic arborization (da) neurons. Similar to sensory neurons in the 
mammalian dorsal root ganglia (DRG), these da neurons' somas and dendritic branches are located in the periphery, and their axons travel through peripheral tissues and into the ventral nerve cord (VNC), a part of the Drosophila CNS, to form synapses. Based on dendritic branching morphology, these da neurons can be divided into four classes: class I to class IV (Grueber et al. 2002). Class I neurons possess the simplest dendrite pattern, classes II and III have more complex dendritic fields, and class IV neurons have the most vigorous dendritic arborization (Grueber et al. 2002).

Song et al. (2012) found that class IV da neurons are able to regenerate their axons if the lesion occurs in the peripheral portion of the axon. In contrast, the other classes fail to elicit a significant regenerative response after axotomy. This is the case even though class III neurons have a similar dendritic phenotype and are born at the same time as class IV ones, suggesting that regenerative capacity is likely independent of dendritic morphology complexity or developmental timing. Such cell type differences came as a surprise because it has been assumed that all sensory neurons in the periphery are regeneration-competent. On the other hand, it is known that different types of neurons in the mammalian CNS possess distinct regenerative capacities. For example, when a permissive graft is transplanted into the injured rat spinal cord, propriospinal axons can regrow into the graft, whereas corticospinal tract axons cannot (Richardson et al. 1982). The results from Song et al. (2012) suggest that differential regenerative ability can be associated with peripheral as well as CNS neurons.

What could account for such cell type-dependent differences in regenerative ability? In principle, neuronal states, including their regenerative ability, might be specified by gene expression programs. Consistent with this, recent studies have implicated certain transcription factors (such as STAT3 [Smith et al. 2009; Sun et al. 2011], KLFs [Moore et al. 2009; Blackmore et al. 2012], and CREBs [Gao et al. 2004]) and translational regulators (such as mTOR [Park et al. 2008; Liu et al. 2010]) as important regulators of intrinsic regenerative ability in mammalian neurons. In Drosophila da neurons, it is known that certain transcription factors regulate the diversity and complexity of dendrites (Grueber et al. 2003; Parrish et al. 2006). These differentiation programs might also predetermine their regrowth potential. It has been shown that a da neuron's dendritic branch pattern can be altered by manipulating the expression of these factors. For example, ectopic expression of cut increases the dendritic complexity of class I da neurons such that it resembles that of class IV neurons (Grueber et al. 2003). It would be interesting to see whether similar manipulations might also alter their regenerative responses. Future studies in this direction have the potential to identify novel transcriptional regulators of regenerative potential.

Another surprising observation from Song et al. (2012), and another example that counters the assumption that all axons in lower organisms regenerate, is that, even in class IV neurons, little regeneration is induced if the lesion occurs within the portion of the axon that resides in the CNS. Instead, these results suggest that these class IV neurons are similar to mammalian DRG sensory neurons, whose axons regenerate well in the periphery but poorly in the CNS. Despite numerous efforts, the mechanisms that account for the differential responses of DRG peripheral and central branches remain unclear. Future genetic studies on these class IV neurons might provide new insights into this question.

\section{Dendrite regeneration}

In contrast to axotomy, very little is known about neuronal responses to dendrite injury. In mammals, it is known that dendritic remodeling occurs both during development and in the adult (Yuste 2011) and that this remodeling can be regulated by neuronal activity (Trachtenberg et al. 2002; Hofer et al. 2009). However, whether injured dendrites are able to regenerate has not been systematically addressed. A major hurdle for such studies is the diffusivity of dendritic projection patterns. The laser-assisted focal lesioning method, together with transparency of the cuticle in Drosophila larvae (Chang and Keshishian 1996; Sugimura et al. 2003; Stone et al. 2010), makes it feasible to sever single dendrites and monitor their regenerative responses in vivo.

By doing this in type IV da neurons, Song et al. (2012) found that the transected dendrite responds in a binary manner: In some cases, the injured dendrites regenerate new branches from the severed stem to reoccupy denervated space. In other cases, the injured dendrite stalls or retracts, and a neighboring dendrite expands its arborization to occupy the empty field left by the injured one. Although it is unknown what accounts for such an "all or none" response, the refilling of empty space by either regeneration or sprouting indicates that these neurons have substantial growth potential.

Strikingly, dendrite regeneration also occurs in a cell type-specific manner. In contrast to the class IV neurons, da neurons in other classes always fail to regenerate their injured dendrites. Moreover, the territory denervated by dendriotomy is not refilled by neighboring branches as it is in class IV neurons. This consistency in cell type specificity for both axon and dendrite regeneration suggests that a common genetic program controls the growth ability of both axons and dendrites.

An interesting note is that these injury experiments were performed in the larvae at the stages when dendrites are still growing (scaling), albeit at a slower rate. However, even in regeneration-competent class IV neurons, only about half of the injured dendrites are able to regenerate, suggesting that other factors in addition to neuronal growth capacity might be involved in dendritic regenerative responses. For example, it would be interesting to see whether the focal dendriotomy triggers consistent injury signals, such as calcium influx as observed after axotomy (McNeil and Kirchhausen 2005; Kamber et al. 2009; Ghosh-Roy et al. 2010). Nevertheless, the establishment of an efficient dendriotomy procedure should facilitate the investigation of these and other issues related to dendrite regeneration. 
A possible regeneration indicator: microtubule (MT) polarity reversal

In these model systems, precise lesioning and live imaging enable the possibility of monitoring cellular events after injury and during regeneration. This has been manifested in recent findings in C. elegans. Genetic studies identified the DLK-1 (dual leucine zipper-bearing kinase 1) MAP kinase pathway as a positive regulator of axon regeneration in C. elegans (Hammarlund et al. 2009; Yan et al. 2009). In continued efforts to explore the underlying mechanisms, Hammarlund et al. (2009) found that the failure of severed axons to regenerate in dlk mutants appeared to be due to the inability to reform a growth cone. Together with other results, these findings suggest that growth cone regeneration can be a rate-limiting step in axon regeneration.

Growth cone regeneration has been extensively studied in cultured neurons (Bradke and Dotti 2000; Verma et al. 2005; Bradke et al. 2012). It has been shown that an initial response to lesioning is a retrograde wave of MT depolymerization in the proximal segment of the injured neurite (Erez et al. 2007). Thus, transforming such destabilized MTs into dynamic cytoskeletal structures that support neurite extension has been proposed to be critical in growth cone formation (Bradke et al. 2012). A logical step in facilitating this would be to stabilize the MT. Indeed, it has been shown that MT stabilization induced by pharmacological or genetic means appears to promote axon regeneration (Erturk et al. 2007; Stone et al. 2008; Chen et al. 2011; Hellal et al. 2011).

A salient feature of MTs is their polarity. In an axon, plus ends point away from the cell body, and this is critical for transporting different organelles and molecules to axonal terminals. In vertebrates, dendritic MTs have a mixed orientation (Dombeck et al. 2003), but in Drosophila, dendrites show a uniform organization of MTs, with the plus end always pointing toward the cell body (Stone et al. 2008). The question is: What happens to the MT polarity after axotomy or dendriotomy? By following the MT plus end marker EB1-GFP, Song et al. (2012) found an interesting correlation. MT polarity disorganization always accompanies the regenerative response after either axotomy or dendriotomy (Fig. 1). In class IV da neurons, MT polarity is partially reversed after axotomy, leaving a mixed MT organization in the regenerating axon and in the dendrites closest to the severed axon. Even in severed dendrites that fail to regenerate, mixed MT polarity is seen in the neighboring branch that eventually takes over the vacant space (Stone et al. 2010; Song et al. 2012). On the other hand, in injured dendrites that initiate regeneration, the MT reversal is seen only in the regrowing branch. Thus, it appears that MT polarity reversal might be an important step in transforming injury signals into the initiation of regeneration. It will be interesting to find out whether MT polarity reversal also occurs in other neurons and, more importantly, how MT polarity is re-established in a neurite once it initiates regrowth.

\section{MicroRNA (miRNA)-dependent regulation of neuronal regenerative ability}

As mentioned above, both neuron-intrinsic growth ability and the extracellular environment regulate axon regeneration in mammals. Multiple inhibitory molecules present in the lesion site contribute to limiting regenerative growth in the mammalian CNS. In contrast, Schwann cells rich in laminin and other permissive extracellular matrix proteins promote axon regeneration in the PNS. This also appears to be the case in Drosophila, as regenerating axons always grow with glial processes, which likely play supporting roles similar to Schwann cells in mammalian PNS.

On the other hand, neuron-intrinsic regenerative capacities also determine their regenerative outcome after injury. In recent years, several molecular pathways, such as PTEN/mTOR (Park et al. 2008; Liu et al. 2010), SOCS3/STAT3 (Smith et al. 2009), KLFs (Moore et al. 2009; Blackmore et al. 2012), Smad-1 (Parikh et al. 2011), and DLK-1 (Hammarlund et al. 2009; Yan et al. 2009), have been identified as regulators of the intrinsic ability. It appears that the PTEN/mTOR pathway also regulates the regenerative response in Drosophila da neurons (Song et al. 2012). Similar to mammalian CNS neurons, inhibiting PTEN or activating Akt (a key downstream effector after PTEN inhibition) in Drosophila class IV da neurons enhances their regenerative responses to both axotomy and dendriotomy, suggesting that this as an evolutionarily conserved growth program that regulates neuronal regenerative ability. However, it is important to note that activation of the Akt pathway is not sufficient to activate a significant regenerative response in regeneration-incompetent class I da neurons, suggesting that other Akt-independent pathways are also critical in regulating neuronal responses to injury.

Despite the role of Akt/mTOR in regulating neuronal growth potential, how such pathways are regulated during development and after injury remains unsolved. Many different mechanisms have been implicated in regulating mTOR activity in nonneuronal cells. The results by Song et al. (2012) reveal an interesting new player; namely, the miRNA bantam (ban). This miRNA acts non-cell-autonomously to regulate neuronal Akt activity and regenerative potential. Initial hints that this may be true came from a forward genetic screen that searched for molecules regulating the growth of dendritic arbors of sensory neurons in Drosophila (Parrish et al. 2009). During larval stages, dendrites of da sensory neurons first exhibit a rapid growth phase to establish dendritic coverage of their receptive fields. This is followed by a subsequent scaling phase in which dendrites grow in proportion to the growth of their target epithelial cells and the larva as a whole to maintain receptive field coverage. The miRNA ban was identified as a potent regulator of this dendritic scaling. In ban mutants, dendrites initially establish proper receptive fields, but their growth fails to synchronize with the growth of their epithelial targets during the scaling phase. This then leads to a late stage phase of exuberant dendritic growth. 
A

\section{B \\ AFTER INJURY \\ (Microtubules depolymerization)}

C

PRE-REGENERATION

( Microtubules polarity reversal)

D

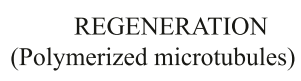

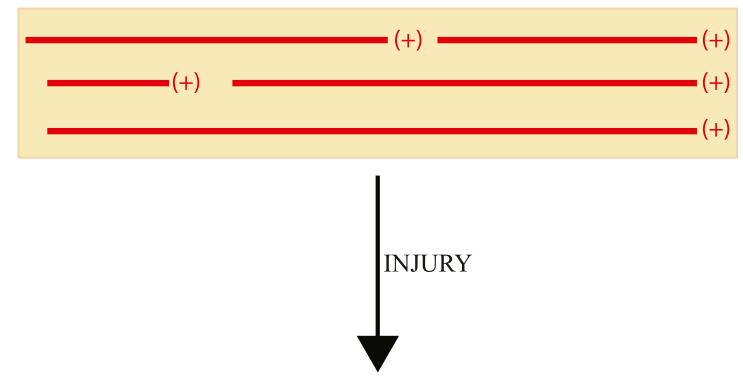
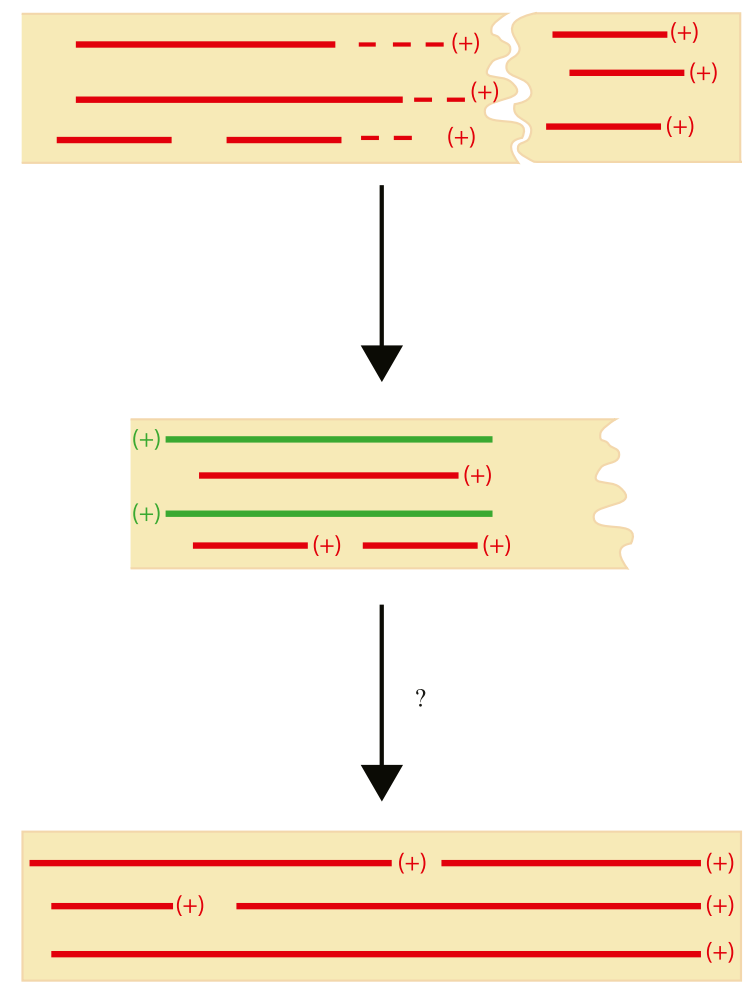

Figure 1. Schematic drawings showing the stages of MT reorganization in an axon leading to a successful regeneration. $(A)$ In an intact axon, MTs (red lines) are polymerized and oriented with the plus end $(+)$ distal to the cell body. $(B)$ After injury, massive MT depolymerization occurs (dashes). (C) In the preregenerative step, there is a MT polarity reversal (green lines) prior to successful regeneration. $(D)$ Once regeneration is initiated, MTs repolarize via unknown mechanisms. Similar events occur after injury in dendrites, except that the polarity is reversed.
Interestingly, instead of acting in da neurons, ban appears to function in their target epithelial cells, and this results in a down-regulation of Akt expression and activity in da neurons by unknown mechanisms. Song et al. (2012) showed that after dendritomy, da neurons in ban mutants exhibited enhanced regeneration, which would correlate with the late stage period of dendritic growth seen in the Parrish et al. (2009) study. Furthermore, this enhanced regeneration could be mimicked by either PTEN silencing or Akt overexpression in neurons. These results reveal a novel miRNA-dependent mechanism by which the dendritic targets can regulate pathways in neurons that control growth potential.

Interestingly, ban's involvement in dendrite regeneration is reminiscent of the role of miR-206, a skeletal muscle-specific miRNA, in promoting the regeneration of the neuromuscular synapses in mice (Williams et al. 2009). Mice that are genetically deficient in miR-206 develop normal neuromuscular synapses but show a defect in regeneration of such synapses after acute nerve injury. Instead of affecting axon regeneration, miR-206 expressed in muscle might generate a local signal that influences the interaction between regenerating motor axons and muscle fibers. Together, these results suggest that miRNAs expressed in the targets of neurons might be a general mechanism regulating neuronal growth ability. Obviously, many questions are still open. For example, how is the expression of these miRNAs regulated? Is the regulation bidirectional between the target and neuron? What are the mediators of miRNA signaling in nonneuronal cells as well as neurons? Also, how do such miRNA-dependent mechanisms affect neuronal regenerative ability in other neurons, including those in the mammalian CNS?

Small invertebrates such as C. elegans and Drosophila melanogaster have become powerful model systems for deciphering the complex genetic networks and mechanisms involved in axon regeneration by virtue of the ability to perform forward and reverse genetic manipulations in these systems. They also present a significant advantage compared with mammals for live imaging. Because their cuticle is transparent, it is possible to 
perform real-time imaging of a single axon within the animal to study axonal regrowth and the underlying processes after injury. Undoubtedly, these organisms will continue to complement mammalian models and reveal important insights into the cellular and molecular mechanisms of axon and dendrite regeneration.

\section{Acknowledgments}

We apologize to our colleagues whose relevant work was not discussed due to space limitations. K.Z. is supported by a postdoctoral fellowship from the Craig H. Neilsen Foundation.

\section{References}

Bejiani RE, Hammarlund M. 2012. Notch signaling inhibits axon regeneration. Neuron 73: 268-278.

Blackmore MG, Wang Z, Lerch JK, Motti D, Zhang YP, Shields CB, Lee JK, Goldberg JL, Lemmon VP, Bixby JL. 2012. Kruppel-like Factor 7 engineered for transcriptional activation promotes axon regeneration in the adult corticospinal tract. Proc Natl Acad Sci 109: 7517-7522.

Bradke F, Dotti CG. 2000. Differentiated neurons retain the capacity to generate axons from dendrites. Curr Biol 10: 1467-1470.

Bradke F, Fawcett JW, Spira ME. 2012. Assembly of a new growth cone after axotomy: The precursor to axon regeneration. Nat Rev Neurosci 13: 183-193.

Chang TN, Keshishian H. 1996. Laser ablation of Drosophila embryonic motoneurons causes ectopic innervation of target muscle fibers. J Neurosci 16: 5715-5726.

Chen L, Wang Z, Ghosh-Roy A, Hubert T, Yan D, O'Rourke S, Bowerman B, Wu Z, Jin Y, Chisholm AD. 2011. Axon regeneration pathways identified by systematic genetic screening in C. elegans. Neuron 71: 1043-1057.

Dombeck DA, Kasischke KA, Vishwasrao HD, Ingelsson $\mathrm{M}$, Hyman BT, Webb WW. 2003. Uniform polarity microtubule assemblies imaged in native brain tissue by second-harmonic generation microscopy. Proc Natl Acad Sci 100: 7081-7086.

Erez H, Malkinson G, Prager-Khoutorsky M, De Zeeuw CI, Hoogenraad CC, Spira ME. 2007. Formation of microtubulebased traps controls the sorting and concentration of vesicles to restricted sites of regenerating neurons after axotomy. J Cell Biol 176: 497-507.

Erturk A, Hellal F, Enes J, Bradke F. 2007. Disorganized microtubules underlie the formation of retraction bulbs and the failure of axonal regeneration. J Neurosci 27: 9169-9180.

Fitch MT, Silver J. 2008. CNS injury, glial scars, and inflammation: Inhibitory extracellular matrices and regeneration failure. Exp Neurol 209: 294-301.

Gao Y, Deng K, Hou J, Bryson JB, Barco A, Nikulina E, Spencer T, Mellado W, Kandel ER, Filbin MT. 2004. Activated CREB is sufficient to overcome inhibitors in myelin and promote spinal axon regeneration in vivo. Neuron 44: 609-621.

Ghannad-Rezaie M, Wang X, Mishra B, Collins C, Chronis N. 2012. Microfluidic chips for in vivo imaging of cellular responses to neural injury in Drosophila larvae. PLOS ONE 7: e29869. doi: 10.1371/journal.pone.0029869.

Ghosh-Roy A, Wu Z, Goncharov A, Jin Y, Chisholm AD. 2010. Calcium and cyclic AMP promote axonal regeneration in Caenorhabditis elegans and require DLK-1 kinase. I Neurosci 30: 3175-3183.

Grueber WB, Jan LY, Jan YN. 2002. Tiling of the Drosophila epidermis by multidendritic sensory neurons. Development 129: $2867-2878$.
Grueber WB, Jan LY, Jan YN. 2003. Different levels of the homeodomain protein cut regulate distinct dendrite branching patterns of Drosophila multidendritic neurons. Cell 112: 805-818.

Guo SX, Bourgeois F, Chokshi T, Durr NJ, Hilliard MA, Chronis N, Ben-Yakar A. 2008. Femtosecond laser nanoaxotomy lab-on-a-chip for in vivo nerve regeneration studies. Nat Methods 5: 531-533.

Hammarlund M, Nix P, Hauth L, Jorgensen EM, Bastiani M. 2009. Axon regeneration requires a conserved MAP kinase pathway. Science 323: 802-806.

Hellal F, Hurtado A, Ruschel J, Flynn KC, Laskowski CJ, Umlauf M, Kapitein LC, Strikis D, Lemmon V, Bixby J, et al. 2011. Microtubule stabilization reduces scarring and causes axon regeneration after spinal cord injury. Science 331: 928-931.

Hofer SB, Mrsic-Flogel TD, Bonhoeffer T, Hubener M. 2009. Experience leaves a lasting structural trace in cortical circuits. Nature 457: 313-317.

Kamber D, Erez H, Spira ME. 2009. Local calcium-dependent mechanisms determine whether a cut axonal end assembles a retarded endbulb or competent growth cone. Exp Neurol 219: 112-125.

Krause TL, Fishman HM, Ballinger ML, Bittner GD. 1994. Extent and mechanism of sealing in transected giant axons of squid and earthworms. J Neurosci 14: 6638-6651.

Liu K, Lu Y, Lee JK, Samara R, Willenberg R, Sears-Kraxberger I, Tedeschi A, Park KK, Jin D, Cai B, et al. 2010. PTEN deletion enhances the regenerative ability of adult corticospinal neurons. Nat Neurosci 13: 1075-1081.

Liu K, Tedeschi A, Park KK, He Z. 2011. Neuronal intrinsic mechanisms of axon regeneration. Annu Rev Neurosci 34: 131-152.

Martin SM, O'Brien GS, Portera-Cailliau C, Sagasti A. 2010. Wallerian degeneration of zebrafish trigeminal axons in the skin is required for regeneration and developmental pruning. Development 137: 3985-3994.

McNeil PL, Kirchhausen T. 2005. An emergency response team for membrane repair. Nat Rev Mol Cell Biol 6: 499-505.

Moore DL, Blackmore MG, Hu Y, Kaestner KH, Bixby JL, Lemmon VP, Goldberg JL. 2009. KLF family members regulate intrinsic axon regeneration ability. Science 326: 298-301.

O'Brien GS, Martin SM, Sollner C, Wright GJ, Becker CG, Portera-Cailliau C, Sagasti A. 2009. Developmentally regulated impediments to skin reinnervation by injured peripheral sensory axon terminals. Curr Biol 19: 2086-2090.

Parikh P, Hao Y, Hosseinkhani M, Patil SB, Huntley GW, Tessier-Lavigne M, Zou H. 2011. Regeneration of axons in injured spinal cord by activation of bone morphogenetic protein/Smad1 signaling pathway in adult neurons. Proc Nat1 Acad Sci 108: E99-E107. doi: 10.1073/pnas.1100426108.

Park KK, Liu K, Hu Y, Smith PD, Wang C, Cai B, Xu B, Connolly L, Kramvis I, Sahin M, et al. 2008. Promoting axon regeneration in the adult CNS by modulation of the PTEN/ mTOR pathway. Science 322: 963-966.

Parrish JZ, Kim MD, Jan LY, Jan YN. 2006. Genome-wide analyses identify transcription factors required for proper morphogenesis of Drosophila sensory neuron dendrites. Genes Dev 20: 820-835.

Parrish JZ, Xu P, Kim CC, Jan LY, Jan YN. 2009. The microRNA bantam functions in epithelial cells to regulate scaling growth of dendrite arbors in Drosophila sensory neurons. Neuron 63: 788-802.

Richardson PM, McGuinness UM, Aguayo AJ. 1982. Peripheral nerve autografts to the rat spinal cord: Studies with axonal tracing methods. Brain Res 237: 147-162. 
Schwab ME. 2010. Functions of Nogo proteins and their receptors in the nervous system. Natl Rev 11: 799-811.

Smith PD, Sun F, Park KK, Cai B, Wang C, Kuwako K, MartinezCarrasco I, Connolly L, He Z. 2009. SOCS3 deletion promotes optic nerve regeneration in vivo. Neuron 64: 617-623.

Song Y, Ori-McKenney KM, Zheng Y, Han C, Jan LY, Jan YN. 2012. Regeneration of Drosophila sensory neuron axons and dendrites is regulated by the Akt pathway involving Pten and microRNA bantam. Genes Dev (this issue). doi: 10.1101/ gad.193243.112.

Spira ME, Oren R, Dormann A, Gitler D. 2003. Critical calpaindependent ultrastructural alterations underlie the transformation of an axonal segment into a growth cone after axotomy of cultured Aplysia neurons. I Comp Neurol 457: 293-312.

Stone MC, Roegiers F, Rolls MM. 2008. Microtubules have opposite orientation in axons and dendrites of Drosophila neurons. Mol Biol Cell 19: 4122-4129.

Stone MC, Nguyen MM, Tao J, Allender DL, Rolls MM. 2010. Global up-regulation of microtubule dynamics and polarity reversal during regeneration of an axon from a dendrite. Mol Biol Cell 21: 767-777.

Sugimura K, Yamamoto M, Niwa R, Satoh D, Goto S, Taniguchi M, Hayashi S, Uemura T. 2003. Distinct developmental modes and lesion-induced reactions of dendrites of two classes of Drosophila sensory neurons. I Neurosci 23: 37523760.

Sun F, Park KK, Belin S, Wang D, Lu T, Chen G, Zhang K, Yeung C, Feng G, Yankner BA, et al. 2011. Sustained axon regeneration induced by co-deletion of PTEN and SOCS3. Nature 480: 372-375.

Trachtenberg JT, Chen BE, Knott GW, Feng G, Sanes JR, Welker E, Svoboda K. 2002. Long-term in vivo imaging of experiencedependent synaptic plasticity in adult cortex. Nature 420: 788-794.

Verma P, Chierzi S, Codd AM, Campbell DS, Meyer RL, Holt CE, Fawcett JW. 2005. Axonal protein synthesis and degradation are necessary for efficient growth cone regeneration. I Neurosci 25: 331-342.

Williams AH, Valdez G, Moresi V, Qi X, McAnally J, Elliott JL, Bassel-Duby R, Sanes JR, Olson EN. 2009. MicroRNA-206 delays ALS progression and promotes regeneration of neuromuscular synapses in mice. Science 326: 1549-1554.

Yan D, Wu Z, Chisholm AD, Jin Y. 2009. The DLK-1 kinase promotes mRNA stability and local translation in C. elegans synapses and axon regeneration. Cell 138: 1005-1018.

Yanik MF, Cinar H, Cinar HN, Chisholm AD, Jin Y, Ben-Yakar A. 2004. Neurosurgery: Functional regeneration after laser axotomy. Nature 432: 822.

Yiu G, He Z. 2006. Glial inhibition of CNS axon regeneration. Nat Rev Neurosci 7: 617-627.

Yuste R. 2011. Dendritic spines and distributed circuits. Neuron 71: 772-781. 


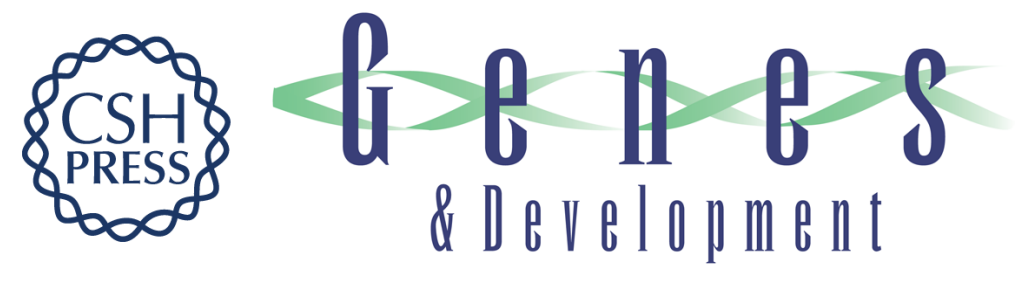

\section{No simpler than mammals: axon and dendrite regeneration in Drosophila}

Homaira Nawabi, Katherine Zukor and Zhigang He

Genes Dev. 2012, 26:

Access the most recent version at doi:10.1101/gad.198150.112
Related Content Regeneration of Drosophila sensory neuron axons and dendrites is regulated by the Akt pathway involving Pten and microRNA bantam
Yuanquan Song, Kassandra M. Ori-McKenney, Yi Zheng, et al.
Genes Dev. July , 2012 26: 1612-1625
References This article cites 46 articles, 19 of which can be accessed free at:
http://genesdev.cshlp.org/content/26/14/1509.full.html\#ref-list-1
Articles cited in:
http://genesdev.cshlp.org/content/26/14/1509.full.html\#related-urls

\section{License}
Email Alerting
Receive free email alerts when new articles cite this article - sign up in the box at the top Service

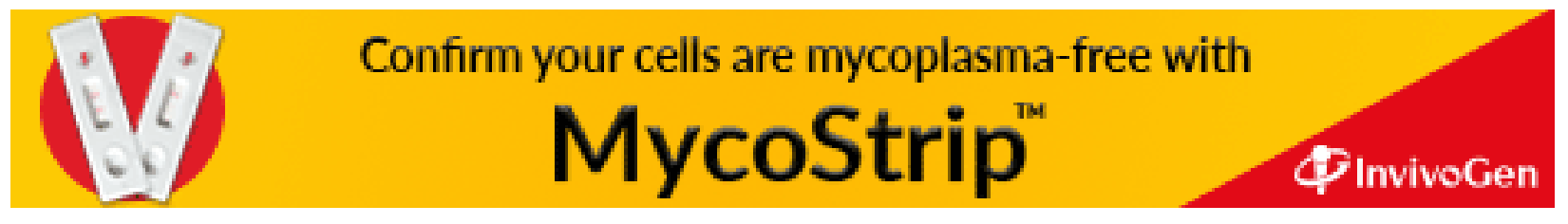

